\title{
Assessment of complex mental activity across the lifespan: development of the Lifetime of Experiences Questionnaire (LEQ)
}

\author{
MICHAEL J. VALENZUELA* AND PERMINDER SACHDEV \\ School of Psychiatry, University of New South Wales, NSW, Australia; The Neuropsychiatric Institute, \\ Prince of Wales Hospital, NSW, Australia
}

\begin{abstract}
Background. Brain reserve is a property of the central nervous system related to complex mental activity which may mediate the course and clinical expression of brain injury. Since there is no instrument that comprehensively assesses complex mental activity through the lifespan, we developed and tested the Lifetime of Experiences Questionnaire (LEQ) in a prospective study of healthy ageing.
\end{abstract}

Method. The LEQ assesses educational, occupational and cognitive lifestyle activities at different stages through life. Test-retest, item analysis and Item Response Theory (IRT) were used to determine reliability. Dimensionality was evaluated using factor analysis. Validity was established through IRT analysis of test performance, correlation with an extant contemporaneous instrument (Cognitive Activities Scale; CAS) and prediction of global cognitive change over 18 months controlling for age, baseline cognition and hypertension.

Results. In a sample of healthy older individuals $(n=79)$ the LEQ was found to be consistent, coherent and discriminate between individuals with high and low mental activity levels. Factor analysis revealed a dominant factor which loaded heavily on education, occupation and leisure activity. Total LEQ was significantly correlated with the CAS. Furthermore, individuals with higher LEQ scores showed less cognitive decline over 18 months, independent of covariates $(r=0 \cdot 37, p=0 \cdot 003)$.

Conclusions. The LEQ is a reliable and valid instrument for assessing complex lifespan mental activity which is protective against cognitive decline. The LEQ is therefore proposed as a useful tool for estimating brain reserve in older individuals and further development is anticipated.

\section{INTRODUCTION}

Brain reserve is a property of the central nervous system related to complex mental activity and hypothesized to alter the course and clinical expression of brain pathology. We have recently conducted a meta-analysis of 22 cohort studies and found that high levels of lifespan complex mental activity was associated with an

\footnotetext{
* Address for correspondence: Dr Michael J. Valenzuela, The Neuropsychiatric Institute, Prince of Wales Hospital, Randwick, NSW 2031, Australia.

(Email: michaelv@unsw.edu.au)
}

almost halved dementia incidence (Valenzuela \& Sachdev, 2006a); systematic review of an independent set of cognitive ageing studies found this association was probably due to attenuated cognitive decline (Valenzuela \& Sachdev, $2006 b$ ). Brain reserve is therefore likely to be a significant factor in the development of dementia in older individuals.

In the past, investigators have assessed behavioural proxies for brain reserve such as education, occupational status and engagement in active lifestyles. Over 15 population-based cohort studies have studied education as a 
dementia risk factor. Meta-analysis showed that the overall odds ratio (OR) for developing dementia related to higher levels of education alone was 0.53 (Valenzuela \& Sachdev, 2006a). The usual method of assessing education has been self-report of the number of years of schooling and then dichotomization into those with more or less than 7-8 years of formal education.

Occupational status has also been investigated as a brain reserve indicator. Stern and colleagues (Stern et al. 1994) found those with higher occupational status were at 0.46 times the risk compared to lower status. Their method of occupational classification was simple: high status included managerial, professional and technical occupations and low status included unskilled, semi-skilled, trade and clerical occupations. Various occupational classifications have since been used, with the main strategies being dichotomization or applying socioeconomic classification systems native to the authors' countries. Results from meta-analysis found heavily in favour of high status occupations leading to a reduced risk of dementia, with 9 out of 12 studies confirming this finding (OR 0.56; Valenzuela \& Sachdev, 2006a).

More recently, engagement in a mentally active lifestyle well into late life has been identified as an important brain reserve factor (Fabrigoule et al. 1995; Fratiglioni et al. 2000; Scarmeas et al. 2001; Fabrigoule, 2002; Verghese et al. 2003). A US study of 801 catholic clergy, unique for employing a validated measure of lifestyle cognitive activity (Cognitive Activities Scale, CAS; Wilson et al. 2002), asked individuals to rate frequency of participation in seven activities for which information processing was considered a central component. CAS scores were inversely correlated to dementia incidence: those with higher scores were at 0.67 times the risk for developing dementia after controlling for age, gender and education. Meta-analysis of the area produced similar results: six studies have confirmed the positive relationship between ongoing mental activity and a lower risk of dementia (OR 0.50; Valenzuela \& Sachdev, 2006a).

Whilst the epidemiological evidence for brain reserve and dementia risk is consistent, there remain a number of limitations in the way the construct has been assessed. (i) Indicators have been looked at in isolation, or at most, in tandem.

(ii) The scope and range of the mental activities have been quite limited. Artistic, sporting, social life, hobbies, reading, learning languages and other mental activities have, for example, not been measured outside of the context of the individual's current lifestyle.

(iii) Indicators have been assessed in a single 'snapshot' fashion. For example, when enquiring about occupational status, only the highest occupational status has been considered.

(iv) With few exceptions, the psychometric properties of the behavioural activity data acquired have not been tested or reported.

The aim of this study was, therefore, to bring together several aspects of epidemiological and behavioural brain reserve research into a new and more comprehensive instrument for psychometric appraisal. Ultimately, validation involved testing the relationship between scores on the scale with incident cognitive change over 18 months after careful control for covariates.

\section{METHOD}

\section{Participants}

Healthy individuals in the control arm of the Sydney Stroke Study participated. Details of the recruitment, selection and assessment processes are available elsewhere (Sachdev et al. 2004a). Briefly, 103 unpaid healthy volunteers aged $>60$ years were recruited during the period 1997-2000. At entry each individual underwent a comprehensive neuropsychological assessment, medical review, psychiatric and psychosocial interview as well as magnetic resonance imaging (MRI). Each of these evaluations (except MRI) was repeated between 12 and 18 months following the initial baseline set of assessments. Ninety-six of the original 103 who entered the study were available for follow-up data collection. Study design was reviewed by the Prince of Wales Hospital Ethics Committee and informed consent was obtained from all participants. The Lifetime of Experience Questionnaire (LEQ) was posted to all 103 control participants following their initial assessment 
and completed questionnaires were mailed back to the primary author.

\section{The LEQ}

\section{Development}

As reviewed in the Introduction and prior reports, behavioural characteristics of brain reserve include participation in mental activities such as education, complex occupations and cognitively stimulating lifestyle activities. The LEQ was therefore developed to comprehensively assess these domains across the lifespan. Accordingly, the LEQ can be said to benefit from a high degree of clinical face validity.

The full LEQ consists of 42 questions, requiring a mix of 5-point Likert scale and free responses about the frequency and intensity of participation on a given activity. Free responses were recoded into an ordinal scale scores on the basis of structured scoring principles as detailed in the Appendix (available online). The LEQ takes approximately 30 minutes to complete.

\section{Structure}

The LEQ was constructed around two major dimensions: (i) Life-stages and (ii) Specific versus non-specific mental activity. LEQ items are available on request from the primary author.

The three life-stages assessed by the LEQ include:

(i) Young Adulthood (13-30 years),

(ii) Mid Life (30-65 years or until retirement),

(iii) Late life (from 65 years or retirement until present date).

Each life-stage section also contained questions that were specific or non-specific to that phase of life. Specific age cut-offs were arbitrary, however, they followed a commonsense approach.

\section{Specific life-stage questions}

In the Young Adulthood section, life-stage specific questions were related to the extent and nature of educational experiences. Postsecondary school courses that took longer or were intellectually more complex were rated higher. Individuals who returned to formal education during Mid Life or Late Life received the equivalent amount of points to their LEQ aggregate score as if they had completed the course in Young Adulthood.

In the Mid Life section, life-stage specific questions were focused on a complete occupational history, with each occupation classified into one of nine categories based on the Australian Standard Classification of Occupations (ASCO; Australian Bureau of Statistics, 1997). Each occupation's managerial capacity was also assessed by asking how many employees or colleagues they were in charge of.

In the Late Life section, life-stage specific questions centred on the person's ongoing social and intellectual activity on a day-to-day basis activities were listed and participants had to rate how frequently they participated in them.

\section{Non-specific mental activity questions}

Respondents were also asked about their participation in a number of non-specific mental activities during each of the three life-stages. These included questions about frequency or proficiency related to: playing musical instruments, following an artistic pursuit, reading, visiting family, social outings, playing sport or practising a physical discipline, learning a new language, travel and hobbies.

\section{Subscores}

The structure of the LEQ permitted the following life-stage subscales to be constructed.

- Young Adulthood score (33.3\% weighting): sum of

- Stage-specific subscore (Education), and

- Non-specific subscore.

- Mid Life score (33.3\% weighting): sum of

- Stage-specific subscore (Occupation and Managerial history), and

- Non-specific subscore.

- Late Life score (33.3\% weighting): sum of

- Stage-specific subscore (Current Social and Intellectual Activity), and

- Non-specific subscore.

The total LEQ was the sum of the three lifestage scores. The specific system used for scoring the LEQ is available in the Appendix (available online). 


\section{Cognitive assessment}

The neuropsychological battery comprised the following tests pertaining to various cognitive domains: verbal memory (Logical Memory I and II subtests from the Wechsler Memory Scale - Revised; Ivnik et al. 1992); visual memory (Visual Reproduction I \& II from WMS-R); working memory [Digit Span backwards, Arithmetic from the Wechsler Adult Intelligence Scale Revised (WAIS-R)]; attention [Digit Span forwards (WAIS-R)]; Mental Control (WMS-R); language (15-item Boston Naming Test); information processing speed (Trail Making Test Part A, Symbol Digit Modalities Test); visuoconstruction [Block Design (WAIS-R) and copying simple figures]; praxisgnosis [Western Aphasia Battery (WAB) ideomotor apraxia subtest items, finger gnosis and stereognosis]; abstract reasoning [Similarities, Picture Completion (WAIS-R)]; mental flexibility (Colour Form Sorting Text, Trail Making Test Part B); Verbal fluency; phonemic (FAS) and semantic (animals). Domain scores were computed at baseline and follow-up as the average $z$ score on relevant tests using baseline norms as the reference point as previously reported (Sachdev et al. 2004b). Trained clinical psychologists performed the assessments.

For the purposes of simplicity, a summary neuropsychological measure was used as the main dependent variable and was calculated as the first principal component extracted from the baseline battery of neuropsychological tests that all participants completed. This summary measure represents $43.5 \%$ of variance in cognitive performance and its use had been reported previously (Sachdev et al. 2004b). Baseline structure coefficients were used to construct the follow-up first principal component analysis (PCA1) score and change in this score calculated by the formula:

$$
\text { PCA1 change }=\mathrm{PCA}_{18 \text { months }}-\mathrm{PCA}_{\text {baseline }} .
$$

\section{Analysis}

Normative LEQ information was developed based on our sample of healthy elderly individuals. Internal coherence was estimated using Cronbach's $\alpha$ test procedure, which calculates the average correlation coefficient between all possible pairs of items in a scale or subscale, using the item analysis tool of SPSS version 14.0 (SPSS Inc., Chicago, IL, USA). Coherence measures were also available from reliability plots derived from Item Response Theory (IRT) analysis (see below for more details). Test-retest consistency was evaluated on a random subsample of 20 control individuals who were asked to complete the LEQ between 3 and 6 months after the first completion using the intra-class correlation procedure. A different random subsample of 20 individuals was additionally interviewed in person in their home and answers from the earlier life-stages (young adulthood and midlife years) explored in depth. For each individual, a document was requested for viewing to corroborate an autobiographical response to a LEQ question.

The assumption of an underlying latent variable was tested using factor analysis. The expectation was that the first principal factor would not only explain the most variance, but also exhibit a large difference in relative eigenvalue when compared to the second factor and that it would include items with loading $>0.3$ that sampled a broad range of complex mental activities.

Validity for the LEQ was then tested in three ways. First, by using IRT. IRT is a non-classical approach to testing the relationship between an individual's performance on particular scale items and proficiency in the underlying latent variable of interest (Bollen, 2002). IRT has been used in the development and analysis of a number of psychological scales (Kahler et al. 2003; Santor et al. 1994). The underlying assumption of IRT is that individuals that are high on a given latent variable $(\theta)$ will have a higher probability of responding positively to a relevant item $[P(\theta)]$ : the relationship between these two measures forms the basis for IRT analysis in the option characteristic curve (OCC).

We used TestGraf software (Ramsay, 2000) for graphical IRT modelling of LEQ test performance. TestGraf begins by ranking respondents on sums of all items and converting these ranks into a standard normal score. This standard normal score is used as a metric for the underlying trait, and probabilities of endorsing a given item calculated to produce the OCC after Gaussian smoothing. Smoothing means that the impact of error surrounding the 
Table 1. Descriptive and item analysis results of raw Lifetime of Experiences Questionnaire (LEQ) subscores in a healthy elderly sample $(n=79)$

\begin{tabular}{|c|c|c|c|c|c|c|c|}
\hline & \multicolumn{2}{|c|}{ Young Adulthood subscore } & \multicolumn{2}{|c|}{ Mid Life subscore } & \multicolumn{2}{|c|}{ Late life subscore } & \multirow[b]{2}{*}{ Total LEQ } \\
\hline & $\begin{array}{l}\text { Stage- } \\
\text { specific }\end{array}$ & $\begin{array}{l}\text { Non-stage- } \\
\text { specific }\end{array}$ & $\begin{array}{l}\text { Stage- } \\
\text { specific }\end{array}$ & $\begin{array}{l}\text { Non-stage- } \\
\text { specific }\end{array}$ & $\begin{array}{l}\text { Stage- } \\
\text { specific }\end{array}$ & $\begin{array}{l}\text { Non-stage- } \\
\text { specific }\end{array}$ & \\
\hline Mean & $11 \cdot 8$ & $12 \cdot 7$ & $12 \cdot 9$ & $12 \cdot 9$ & $12 \cdot 2$ & $11 \cdot 2$ & $75 \cdot 5$ \\
\hline S.D. & $5 \cdot 8$ & $5 \cdot 0$ & $5 \cdot 1$ & $4 \cdot 6$ & $2 \cdot 6$ & $4 \cdot 6$ & $20 \cdot 0$ \\
\hline $\begin{array}{l}\text { Alpha reliability } \\
\text { coefficient }\end{array}$ & 0.43 & $0 \cdot 78$ & $0 \cdot 78$ & $0 \cdot 58$ & $0 \cdot 84$ & $0 \cdot 63$ & $0 \cdot 66$ \\
\hline $\begin{array}{l}\text { Intra-class test- } \\
\text { retest correlation }\end{array}$ & $0.95 *$ & $0.96^{*}$ & $0.99 *$ & $0.96^{*}$ & $0.94 *$ & $0.97 *$ & $0.98^{*}$ \\
\hline
\end{tabular}

Test-retest results were based on a random subsample of $n=20$ who completed LEQ on two occasions 3-6 months apart.

* $p$ values $<0 \cdot 0001$.

original sums of items is considerably reduced (Ramsay, 1991). The point on the OCC at which one item's response option becomes more likely than another (e.g. $>50 \%$ ) can then be used as an index of the difficulty of the item; the steepness of the OCC reflects how well the item discriminates between individuals that are high or low on the underlying trait. The central advantage of the IRT approach is generation of unbiased OCC and reliability plots across the full spectrum of underlying trait levels, rather than assuming a fixed item performance-trait relationship. Furthermore, TestGraf produces relative credibility plots for each individual, in which the maximum-likelihood value depicts a more accurate estimate of the person's true ability level than simply the sum of items because responses to items considered more difficult (at the group level) are weighted more heavily. Finally, TestGraf also produces an Information function, which measures the degree to which items were generally informative in the sense of discriminating between individuals with high and low latent trait proficiency levels (i.e. OCC slope) in relation to the precision of the latent trait estimate $\left[\sigma_{\mathrm{e}}(\theta)\right]$.

The second validation approach tested for a positive correlation between the LEQ and a validated measure of contemporaneous complex mental activity (CAS; Wilson et al. 2002). The third strategy involved determining the correlation between LEQ scores and change on a summary neuropsychological measure over the course of 18 months after control for appropriate confounds. This latter test formed the core validation procedure of the study, with the expectation that higher LEQ scores would be associated with less cognitive decline.

\section{RESULTS}

\section{Normative LEQ data}

Response rate

Of the 103 control individuals who were sent LEQs, 86 were returned, a response rate of $83 \%$. Of these, seven were inadequately completed and were excluded from the analysis, leaving a sample of 79 , giving a final response rate of $77 \%$.

\section{Demographics}

The average age of the sample was 70.9 years (range 58-93) and 56.8\% were male. The average MMSE score was 28.2 (23-30) and the mean NART(r)-IQ score was 114.8 (83-129). $35 \cdot 8 \%$ of participants were hypertensive.

\section{Distribution}

Table 1 shows the average scores and standard deviations on the different LEQ Life-stage subscores and Total LEQ for the cohort. The total LEQ score distribution approximated a normal Gaussian pattern.

\section{Internal coherence}

Item analysis

Results from classical $\alpha$ (Cronbach model) reliability analysis are shown in Table 1. Most of the LEQ Life Stage subscales demonstrated internal coherence $\alpha$ coefficients $>0 \cdot 7$.

The Young Adulthood specific scale was exceptional for having a low $\alpha$ coefficient $(0 \cdot 43)$. 


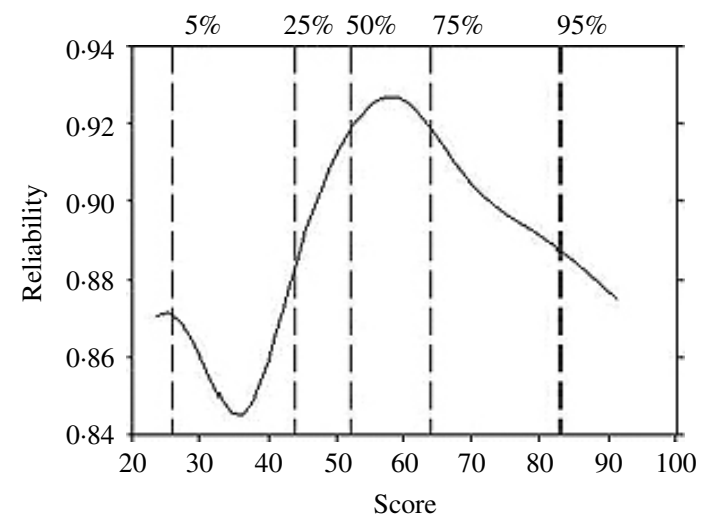

FIG. 1. Reliability function calculated using a non-parametric Item Response Theory approach, showing peak reliability in the mid range of underlying trait levels, moderate reliability at the high end, and lower reliability at the low end of the trait distribution.

These items essentially asked the individual: (i) number of years of high school completed, (ii) if they had gained an end of high school certificate, and (iii) the nature and years of tertiary/ post-school education and training they had completed. While parts (i) and (ii) of this subscale were highly correlated $(r=0.69, p<$ 0.0001 ), there was only a minor relationship between high-school education and post-highschool education or training in our sample $(r=0 \cdot 23, p=0 \cdot 037)$. Thus the lower $\alpha$ coefficient of the Young Adulthood subscale was mainly due to a low level of coherency between levels of pre- and post-high-school education in our sample.

\section{IRT}

As can be seen in Fig. 1, the LEQ demonstrated a polynomial reliability distribution, with peak reliability at the middle trait proficiency range (approximately 0.92 ), followed by the high proficiency range $(0.89)$, and least in the low proficiency range $(0 \cdot 85)$.

\section{Dimensionality}

Factor analysis was performed on all 42 LEQ items. Twenty factors were identified with eigenvalues $>1.0$, together accounting for $81 \%$ of the variance in LEQ data. In order to facilitate interpretation of the factors, the first six factors are considered in more detail and together explained $44 \%$ of variance in the scale. Table 2 shows those items with the strongest loadings for each factor, whether positive or negative. Factor 1 (14\% variance) loaded heavily on occupational, educational and leisure activities including reading and physical pursuits. This factor accounted for almost twice as much as the second factor.

\section{Subscale independence}

The cross-correlations between the Life Stage subscales showed moderate association: YoungMidlife $(r=0.65, p<0.0001)$; Young-Late life $(r=0.54, p<0.0001)$; Mid-Late life $(r=0 \cdot 26$, $p=0 \cdot 019)$.

\section{Consistency}

Test-retest

Seventeen out of 20 individuals completed the LEQ twice. Test-retest intra-class correlation coefficients are shown in Table 1. The overall level of test-retest reliability was high $(r=0 \cdot 98$, $p<0 \cdot 0001, \mathrm{df}=16$ ).

\section{Documentary verification}

Twenty control participants were interviewed at home regarding their LEQ responses. All but one participant was able to provide documentary verification for a response made to early life events.

\section{Validation strategy}

\section{Construct validity}

Case histories of two individuals considered to be emblematic of high and low lifespan complex mental activity are presented below. Maximum likelihood estimates derived from these individuals are displayed in Fig. 2( $a, b)$ and show that the LEQ was able to distinguish between these individuals with a satisfactory level of precision. The LEQ Information function is also presented in Fig. $2 c$, showing that discrimination in relation to variance was highest in the mid-range of the distribution of proficiencies $(0 \cdot 08)$, followed by high-range proficiencies $(0.06)$, and lowest $(0.04)$ at the low range of proficiencies. For comparative purposes, the peak Information function for the Beck Depression Inventory has been estimated to be approximately $0 \cdot 15$ in the low range of depressive symptoms and about 0.08 in the clinically significant top $5 \%$ of the depressive trait distribution (Ramsay, 2000). 
Case history no. 1. Mrs $\mathrm{R}$ is a 71-year-old female with a total LEQ of 119 (maximumlikelihood value estimate 87 th percentile; see Fig. 2a). She completed 5 years secondary education and went on to receive a teaching diploma in her young adulthood. One of her passions is music, having learnt to play a musical instrument at an early age. In the early part of her mid life she became a mature-age student and completed a B.Arts (Education) part-time and then taught English and History at a local high school for the majority of her working life. Sport and exercise were a prominent part of her routine at every life-stage. At the age of 56 she completed a Masters of Arts and was promoted to Deputy School Principal for the remainder of her professional career. Now retired and living with her husband, she remains extremely active. She is a member of no less than seven social and charity groups, visits friends and family weekly and spends a typical day listening to music, studying, participating in voluntary work, reading, doing crosswords, learning to use the computer and developing income streams. During her lifetime she has learnt to speak four foreign languages and has travelled widely in both Australia and virtually every continent. Mrs R's PCA1 score increased by 7.7 units over the 18-month follow-up period.

Case history no. 2. $\mathrm{Mr} \mathrm{S}$ is a 73-year-old male who had total LEQ of 38 (maximum-likelihood value estimate 35 th percentile; see Fig. $2 b$ ). He has never been more than a casual reader, 'just to get by'. Mr S completed no years of secondary or tertiary education, but was an active sportsman in his young adulthood. His working life included jobs in the government service, catering and driving. Whilst his physical activities dropped off during his mid life, since retiring he has taken up sporting pursuits again. The majority of his life since retirement has been spent living alone. He is an active member of three social or charity groups and sees his friends on a regular basis. His contact with family, however, is infrequent. His typical day is spent watching TV, walking, listening to music, participating in voluntary work, socializing at the local club and occasionally playing bingo. He has travelled twice in his life - once to the UK and once to the USA. Mr S's PCA1 score 
(a)

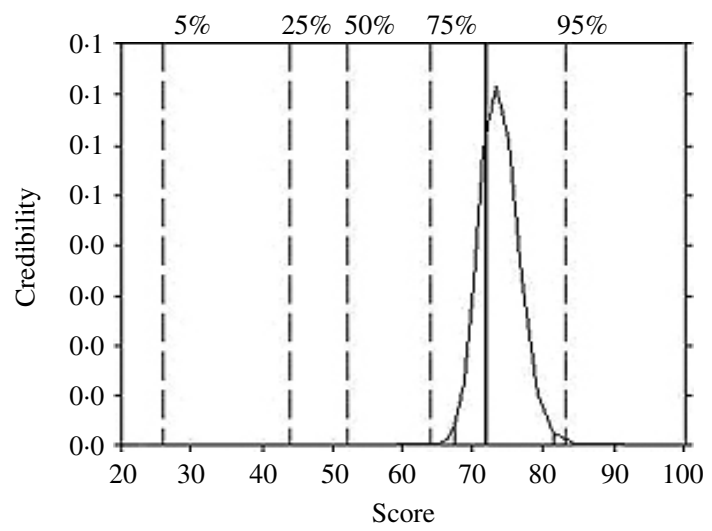

(b)

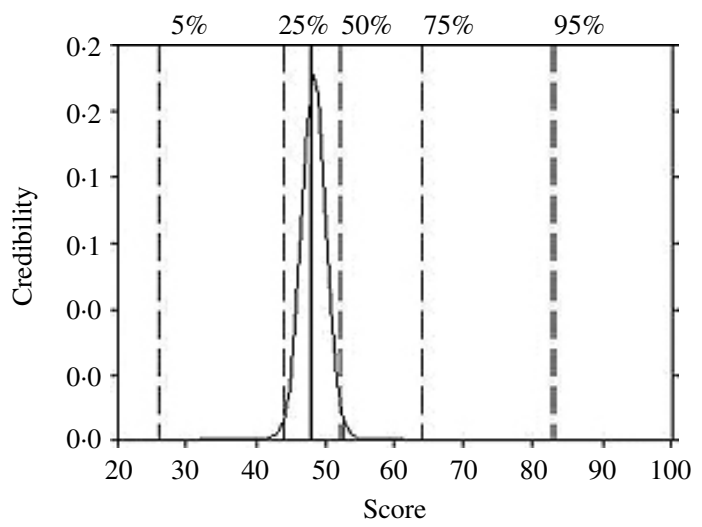

(c)

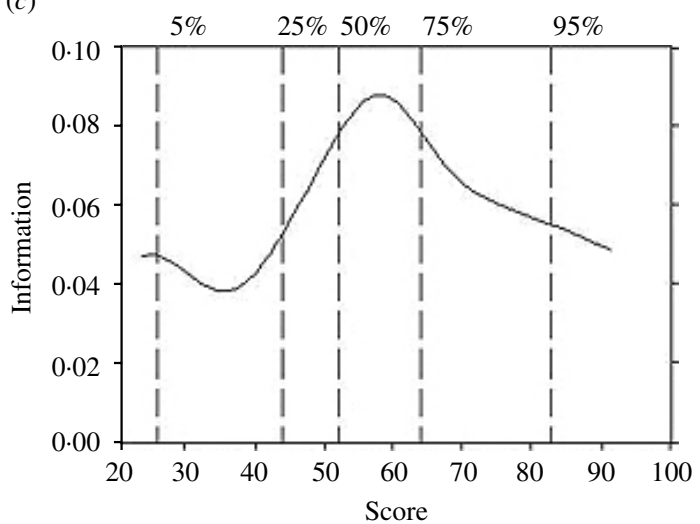

FIG. 2. Maximum likelihood range estimates for complex mental activity proficiency based on Item Response Theory (IRT) weighting of item responses (see text for more details). (a) High mental activity individual presented as Case history no. 1, and $(b)$ low mental activity individual (Case history no. 2). (c) Information function for Lifetime of Experiences Questionnaire (LEQ) based on IRT estimate of general discriminative performance in relation to item variance (see text for more details). dropped by $10 \cdot 7$ units over the 18 -month followup period.

\section{Concurrent validity}

We were able to reconstruct the CAS from identical items in the LEQ and thereby estimate a CAS score. Concurrent validity of the LEQ was demonstrated by a significant zero-order intra-class correlation between total LEQ and CAS $(r=0 \cdot 41, p<0 \cdot 0001, \mathrm{df}=77)$.

\section{Clinical validity: $L E Q$ and cognitive change}

The relationship between the LEQ and prospective cognitive change in PCA1 over 18 months was examined. To facilitate interpretation of subsequent results, the factor structure of PCA1 is provided in Table 3 . Potential confounders including age, baseline cognition and hypertension (Trollor \& Valenzuela, 2001), were controlled for simultaneously. This partial correlation between LEQ and change in PCA1 was $r=0.37 \quad(p=0.003)$. Elimination of covariates did not significantly degrade this finding $(r=$ $0 \cdot 30, p=0 \cdot 017)$. To rule out possible leverage bias effects of extreme values, this analysis was repeated with four outliers excluded, and was unchanged $(r=0 \cdot 37, p=0 \cdot 005)$. Finally, scores derived solely from a particular life- stage were generally found to correlate with PCA1 cognitive change (Young Adulthood partial correlation: $r=0 \cdot 36, p=0.005$; Mid Life partial correlation: $r=0 \cdot 35, p=0.006$; Late Life partial correlation: $r=0 \cdot 22, p=0 \cdot 09)$.

The association between total LEQ score at baseline and change in a number of individual neuropsychological domains was also examined. LEQ was correlated to change in performance in the attention domain $(r=0.32, p=0.01)$, and trends were apparent in the executive $(r=0 \cdot 24$, $p=0.06)$ and working-memory domains $(r=$ $0 \cdot 22, p=0 \cdot 09)$. Correlations in other domains were non-significant.

In addition to testing the association between a priori derived LEQ scores, we also tested the association between cognitive change and datadriven factor scores (after equivalent control for covariates). The first factor $(14 \%$ variance), representing combined educational, occupational experiences and leisure activity, was found to have a significant correlation with change on PCA1 summary performance $(r=$ $0 \cdot 33, p=0 \cdot 012)$. 
Table 3. First principal component analysis (PCA1) factor weightings for individual neuropsychological test scores. PC1 accounted for $43.5 \%$ of variance in cognitive performance at baseline. The same weightings were used to derive the follow-up PCAl score

\begin{tabular}{lc}
\hline \hline Cognitive test & Factor weighting \\
\hline Trail Making Test B & $-0 \cdot 81$ \\
Symbol Digits Modalities & $0 \cdot 81$ \\
Trail Making Test A & $-0 \cdot 79$ \\
Visual Reproduction 1 & $0 \cdot 76$ \\
Block Design & $0 \cdot 75$ \\
Visual Reproduction 2 & $0 \cdot 75$ \\
Arithmetic & $0 \cdot 66$ \\
Animal Naming & $0 \cdot 58$ \\
Similarities & $0 \cdot 57$ \\
Colour Form Sort Test & $0 \cdot 53$ \\
COWAT & $0 \cdot 52$ \\
Digit Symbols & $0 \cdot 49$ \\
Gnosis & $0 \cdot 46$ \\
Drawing & $0 \cdot 42$ \\
Boston Naming Test & $0 \cdot 41$ \\
Logical Memory 2 & $0 \cdot 40$ \\
Logical Memory 1 & $0 \cdot 35$ \\
Ideomotor Apraxia & $0 \cdot 34$ \\
Mental Control & $0 \cdot 22$ \\
\hline \hline
\end{tabular}

\section{DISCUSSION}

The performance characteristics of the LEQ were tested in a sample of 79 healthy elderly participants. Overall, the LEQ appears to reliably measure an individual's lifespan of mental activities and produces several subscores of interest. Moreover, total LEQ was a significant predictor of longitudinal cognitive change after controlling for a number of relevant cofactors, suggesting it measures an underlying neurocognitive phenomenon of potential clinical relevance.

The LEQ's response rate was noted to be high $(77 \%)$, suggesting that the normative information we collected was not overly skewed, or that the instrument was overly taxing. The distribution of scores from our sample also approximated a normal curve, further suggesting no significant bias or inappropriate ceiling or floor effects.

Test-retest reliability was also remarkably high, on average above 0.98 . Each of the subscales demonstrated equivalent stability over a 3- to 6-month period. The internal coherence of items within the different subscales was also mostly adequate, with an overall $\alpha$ coefficient of 0.66. The Young Adult specific subscale, which centred on items related to educational achievement, showed the least coherence. Cohort characteristics of this sample may be partly responsible, because during their young adult years Australia was heavily involved in World War II. The traditional educational pathways we are familiar with now would have been considerably disrupted, and may explain the low correlation between pre- and post-highschool education that was observed.

IRT analysis shed more light on the reliability characteristics of the LEQ. Reliability varied considerably with overall proficiency, with maximal reliability in the mid-range, followed by the high and then low range. These results signify that the current LEQ is well suited to estimating complex mental activity when in the normal and high ranges, but may do less well in the low range. Given that individuals with low-range complex mental activity may be at greater risk for dementia, this is a weakness of the current instrument. Further development will attempt to address this issue by including a greater number of items to which low activity individuals can respond affirmatively.

Factor analysis revealed that the LEQ assesses a multidimensional construct. However, the first factor was dominant in the sense of accounting for almost twice the variance of the next factor, and more significantly, combined items from the educational, occupational and lifestyle domain across the lifespan. The primary dimension that emerged therefore suitably reflected the intent and design of the scale as a whole. The particular LEQ scoring system that was developed also received support from this analysis, because correlation between these a priori scores and longitudinal cognitive change $(r=0.37)$ was similar to the result when using the data-driven Factor 1 score $(r=0 \cdot 33)$.

Assessments of the full range of mental activities at each stage of life were only moderately inter-correlated. The highest correlation was between the Young Adulthood and Mid Life subsections $(0.65)$ and the lowest between Mid Life and Late Life $(0 \cdot 26)$. This result demonstrates the need for a lifespan approach to assessing complex mental activity, since only one facet, e.g. educational achievements in young adulthood, will only mildly predict mental activity in another life-stage. 
Validation of the LEQ was addressed in three ways. First, IRT was used to establish that the items were generally able to discriminate between individuals with high and low complex mental activity with respect to test variance (Information function). The LEQ's Information function peaked at 0.08 in the mid range of scores and dropped to 0.04 in the low range. We compared this to the Information function of a highly regarded scale for assessing depressive mood, the Beck Depression Inventory (Ramsay, 2000), and found the LEQ performed approximately half as well. Overall, this was interpreted favourably given the implicit heterogeneity of complex mental activity in comparison to depressed mood symptoms, and that this is the initial version of an instrument that will have further development.

Second, concurrent validity for the LEQ was demonstrated by positive correlation with a reconstructed version of the CAS (Wilson et al. 2002), a scale unique for being validated in the dementia area. Whilst not an ideal method of test, this result served to confirm that the LEQ estimates complex mental activity in a meaningful and corroborated manner.

A strong third validation challenge was posed in the form of the statement: 'If those with higher behavioural brain reserve show a decreased risk of dementia and a lower rate of cognitive decline, then those with greater LEQ scores should show less cognitive decline over time even after adjusting for known predictors of interest such as age and cardiovascular disease.' Furthermore, in order to eliminate the 'passive' effect of neuropsychological test competence at the start of the study (i.e. ascertainment bias; Gilleard, 1997), baseline cognition was also controlled for (Glei et al. 2005). A positive relationship between total LEQ and cognitive change was found and noted to be insensitive to exclusion of outlier data. Importantly, life-stage subscores showed a similar relationship with cognitive decline as the total LEQ score. The LEQ total score may therefore represent a useful approximation of the neurocognitive brain-reserve construct.

There were, however, limitations to the study. As mentioned, reliability was sub-optimal in the low range of complex mental activity. Our sample was also well above average in intelligence, with an average NART-IQ of 114, almost a full standard deviation above the mean. This was probably due to volunteer control recruitment rather than a random community-based sample, and limits generalization of our normative results. Limited follow-up time also meant that the LEQ's potential clinical utility as a predictor of incident dementia, as opposed to cognitive decline, could not be tested. A community-based test of LEQ would therefore be the next step in further addressing these issues.

The LEQ was demonstrated to be a reliable and valid tool for the estimation of complex mental activity across the lifespan, which probably represents an important protective factor in the context of dementia and human ageing. Further research is anticipated that may lead to development of a useful clinical tool.

\section{ACKNOWLEDGMENTS}

This study was funded by the National Health and Medical Research Council of Australia.

\section{NOTE}

Supplementary material accompanies this paper on the Journal's website (http://journals. cambridge.org).

\section{DECLARATION OF INTEREST}

None.

\section{REFERENCES}

Australian Bureau of Statistics (ABS) (1997). Australian Standard Classification of Occupations (2nd edn). (http://www.abs.gov.au Ausstats/abs@.nsf/525a1b9402141235ca25682000146abc/). Accessed 1 March 2003.

Bollen, K. (2002). Latent variables in psychology and the social sciences. Annual Review of Psychology 53, 605-634.

Fabrigoule, C. (2002). Do leisure activities protect against Alzheimer's disease? Lancet Neurology 1, 11

Fabrigoule, C., Letenneur, L., Dartigues, J. F., Zarrouk, M., Commenges, D. \& Barberger-Gateau, P. (1995). Social and leisure activities and risk of dementia: a prospective longitudinal study. Journal of the American Geriatric Society 43, 485-490.

Fratiglioni, L., Wang, H. X., Ericsson, K., Maytan, M. \& Winblad, B. (2000). Influence of social network on occurrence of dementia: a community-based longitudinal study. Lancet $\mathbf{3 5 5}$, 1315-1319.

Gilleard, C. (1997). Education and Alzheimer's disease: a review of recent international epidemiological studies. Aging and Mental Health 1, 33-46.

Glei, D., Landau, D., Goldman, N., Chuang, Y., Rodriguez, G. \& Weinstein, M. (2005). Participation in social activities helps 
preserve cognitive function: an analysis of a longitudinal, population-based study of the elderly. International Journal of Epidemiology 34, 864-871.

Ivnik, R., Malec, J., Smith, G., Tangalos, E., Petersen, R., Kokmen, E. \& Kurland, L. (1992). Mayo's older Americans normative studies: WMS-R norms for ages 56-94. The Clinical Neuropsychologist 6 (Suppl.), 49-82.

Kahler, C., Strong, D., Hayaki, J., Ramsay, S. \& Brown, R. (2003). An item response analysis of the Alcohol Dependence Scale in treatment-seeking alcoholics. Journal of Studies on Alcohol $\mathbf{1}$, $127-136$.

Ramsay, J. (1991). Kernel smoothing approaches to nonparametric item characteristic curve estimation. Psychometrika 56, 611-630.

Ramsay, J. (2000). TestGraf: a program for the graphical analysis of multiple choice test and questionnaire data. Software manual (details available from: http://www.psych.mcgill.ca/ faculty/ramsay/TestGraf.html).

Sachdev, P., Brodaty, H., Valenzuela, M., Lorentz, L., Looi, J., Wen, W. \& Zagami, A. (2004a). The neuropsychological profile of vascular cognitive impairment in stroke and TIA patients. Neurology 62, 912-919.

Sachdev, P. S., Brodaty, H., Valenzuela, M. J., Lorentz, L. \& Koschera, A. (2004b). Progression of cognitive impairment in stroke patients. Neurology 63, 1618-1624.

Santor, D., Ramsay, J. \& Zuroff, D. (1994). Nonparametric item analyses of the Beck Depression Inventory: evaluating gender item bias and response option weights. Psychological Assessment 6, 255-270.
Scarmeas, N., Levy, G., Tang, M., Manly, J. \& Stern, Y. (2001) Influence of leisure activity on the incidence of Alzheimer's disease. Neurology 57, 2236-2242.

Stern, Y., Gurland, B., Tatemichi, T., Tang, M., Wilder, D. \& Mayeux, R. (1994). Influence of education and occupation on the incidence of Alzheimer's disease. Journal of the American Medical Association 271, 1004-1010.

Trollor, J. \& Valenzuela, M. (2001). Brain ageing in the new millenium. Australian and New Zealand Journal of Psychiatry 35, 788-805.

Tuokko, H., Garrett, D., McDowell, I., Silverberg, N. \& Kristjansson, B. (2003). Cognitive decline in high-functioning older adults: reserve or ascertainment bias? Aging \& Mental Health $\mathbf{7}$, 259-270.

Valenzuela, M. J. \& Sachdev, P. (2006a). Brain reserve and dementia: a systematic review. Psychological Medicine 36, 441-454.

Valenzuela, M. J. \& Sachdev, P. (2006b). Brain reserve and cognitive decline: a nonparametric systematic review. Psychological Medicine 36, 1065-1073.

Verghese, J., Lipton, R., Katz, M., Hall, C., Derby, C., Kuslansky, G., Ambrose, A., Sliwinski, M. \& Buschke, H. (2003). Leisure activities and the risk of dementia in the elderly. New England Journal of Medicine 348, 2508-2516.

Wilson, R. S., Mendes De Leon, C. F., Barnes, L. L., Schneider, J. A., Bienias, J. L., Evans, D. A. \& Bennett, D. A. (2002). Participation in cognitively stimulating activities and risk of incident Alzheimer disease. Journal of the American Medical Association 287, 742-748. 